\title{
Solutions of Hyperbolic Equations with the CIP-BS Method*
}

\author{
Takayuki UTSUMI**, Takashi YABE ${ }^{* * *}$, Takayuki AOKI ${ }^{* * * *}$, \\ James KOGA** $^{* *}$ and Mitsuru YAMAGIWA**
}

\begin{abstract}
In this paper, we show that a new numerical method, the Constrained Interpolation Profile - Basis Set (CIP-BS) method, can solve general hyperbolic equations efficiently. This method uses a simple polynomial basis set that is easily extendable to any desired higherorder accuracy. The interpolating profile is chosen so that the subgrid scale solution approaches the local real solution owing to the constraints from the spatial derivatives of the master equations. Then, introducing scalar products, the linear and nonlinear partial differential equations are uniquely reduced to the ordinary differential equations for values and spatial derivatives at the grid points. The method gives stable, less diffusive, and accurate results. It is successfully applied to the continuity equation, the Burgers equation, the Korteweg-de Vries equation, and one-dimensional shock tube problems.
\end{abstract}

Key Words: the CIP-BS Method, the CIP Method, Basis Set, Hyperbolic Equations, Galerkin Formulation

\section{Introduction}

During the past three decades there has been significant progress in numerical analysis for hyperbolic equations, especially for hydrodynamic equations. We can expect that the recent progress in computer hardware will serve increasing accuracies of numerical solutions to some extent by enabling the use of finer meshes. However, nowadays, it is widely recognized that tracing sharp discontinuities of physical quantities such as shock waves or interfaces between different materials can not be satisfactorily accomplished with this technique. Therefore, there is continuing interest in developing more robust and efficient methods for numerically solving general hyperbolic equations.

There are basically two types of methods for numerical solutions for hyperbolic equations: spectral and grid methods. The main difference between these two methods comes from the methodology in treating the spatial

* Received 16th June, 2004 (No. 04-4157)

** Japan Atomic Energy Research Institute, 8-1 Umemidai Kizu-cho, Souraku-gun, Kyoto 619-0215, Japan. E-mail: utsumi@apr.jaeri.go.jp

*** Department of Mechanical Engineering and Science, Tokyo Institute of Technology, O-okayama, Meguro-ku, Tokyo 152-8552, Japan

**** Global Scientific Information and Computing Center, Tokyo Institute of Technology, O-okayama, Meguro-ku, Tokyo 152-8552, Japan derivatives. Since spectral methods typically consist of expanding the total solution in terms of differentiable basis functions such as used in the Fourier transformation, they do not suffer from numerical inaccuracies for derivatives. Therefore, in general, these methods give accurate solutions with a minimum number of discretization points, only if appropriate problem specific basis functions which satisfy the boundary conditions are applicable. On the other hand, grid methods, such as finite element methods or finite difference methods, consist of approximating derivatives by finite differences. It is often difficult to approximate the derivatives with sufficient accuracy, because the derivatives are estimated by using only the values of the function on a compact set of grid points. However, since finite difference derivatives often lead to structured matrices, which are easily adapted to a number of standard techniques, they are typically more flexible and easier to implement than spectral methods for systems with complex boundary conditions. Therefore, it is to be said that improvements in grid methods which exemplify the spectral method's accuracy is the primary goal of a numerical method.

As far as incorporating only the values at grid points, it seems difficult to achieve the previously stated goal. In 1991, Yabe and Aoki proposed the Constrained Interpolation Profile (CIP) method $^{(1)}$, in which not only values but also their first derivatives are treated as independent variables associated with the grid point, and the information lost inside the grid cell is retrieved by a Hermite type in- 
terpolation function ${ }^{(2)}$. The CIP method belonging to the class of the grid methods has been shown to be a low numerical diffusion and stable method as a universal solver for hyperbolic differential equations ${ }^{(3)}$, and has been successfully applied to various complex linear and nonlinear hydrodynamic problems, covering both compressible and incompressible flow ${ }^{(4)-(7)}$, such as shock wave generation, laser-induced evaporation, and elastic-plastic flow.

It is worth noting that the CIP method does not include any algorithm-dependent parameter. If the size of the system and grid intervals for the problem are defined, the governing equations are uniquely transformed into a discretized form, in which no matrix solution is used. However, methods using matrix operations are more advantageous to investigate the characteristics of the system, and a number of numerical methods for large, sparse systems developed for the finite difference method or the finite element method can be adopted. Recently, a new numerical method, the CIP-Basis Set (CIP-BS) method ${ }^{(8)}$, has been proposed by generalizing the concept of the CIP method from the viewpoint of the basis set. The governing equations are unambiguously discretized into matrix form equations requiring the residuals to be orthogonal to the basis functions via the same procedure as the Galerkin method. The CIP-BS method, in which the local polynomial basis functions corresponding to the values and spatial derivatives at each grid point belong to the complete set and the $\mathrm{C}^{K}$ class, is called the CIP-BS ${ }^{K}$ method. Numerical results in the solution of the Schrödinger equation have demonstrated that accurate solutions are obtained by the CIP-BS method and the use of a higher order basis set is essential in increasing accuracy.

Considering these historical development, the CIPBS method has been explored, it is reasonable to expect the method can be applied to general hyperbolic equations. The purpose of this paper is to show that the CIP-BS method, in which the functional values and their derivatives are associated with grid points having well-defined spectral basis functions, can solve various types of hyperbolic equations with high accuracy and be a universal solver for them by presenting examples for the solutions of typical equations.

The paper is organized as follows: In section 2, we briefly review the CIP-BS method adding extensions to adopt the method to nonlinear hyperbolic equations. In section 3, the discretized equations are explicitly described for typical hyperbolic equations: the continuity, Burgers, Korteweg-de Vries (KdV), and hydrodynamic equation. The efficiency and accuracy of the CIP-BS method are demonstrated by numerical results. Discussions on the multi-dimensional basis set and concluding remarks are given in sections 4 and 5, emphasizing the computational efficiency and applicability of the CIP-BS method for the study of the dynamics of a broad spectrum of complex physical and engineering problems.

\section{Numerical Method}

Since the CIP-BS method is new and not widely known, we first summarize the method adding extensions to adopt the method to nonlinear hyperbolic equations.

We need a basis set where it is easy to define values and derivatives of an arbitrary function, $f(x)$, at the grid points. Therefore, we assume that the functions in the domain of $R^{1}$ can be approximated by the CIP-basis set of degree $K$ method (CIP-BS ${ }^{K}$ ), where $K$ refers to the order of the derivatives we retain in the calculation, through the expression

$$
f(x)=\sum_{i=1}^{N} \sum_{k=0}^{K} f_{i}^{(k)} \phi_{k, i}(x)
$$

where $f_{i}^{(k)}$ is the $k$-th coefficient at the grid point $x_{i}$, the summation on the index $i$ is taken over all grid points, and the basis functions, $\phi_{k, i}(x)$, on the local support $\left[x_{i-1}, x_{i+1}\right]$ are expressed in the form

$$
\phi_{k, i}(x)=\theta_{i-1, i}(x) \phi_{k, i-}(x)+\theta_{i, i+1}(x) \phi_{k, i+}(x),
$$

where $\theta_{i, i+1}(x)=\theta\left(x-x_{i}\right)-\theta\left(x-x_{i+1}\right), \theta(x)$ is the Heaviside step function, and $\phi_{k, i-}(x), \phi_{k, i+}(x)$ are polynomials of degree $(2 K+1)$ determined from the constraints:

$$
\begin{aligned}
& D_{x}^{l} \phi_{k, i \pm}\left(x_{i}\right)= \begin{cases}1 & \text { for } l=k \\
0 & \text { for } l=0,1, \ldots, k-1, k+1, \ldots, K\end{cases} \\
& D_{x}^{l} \phi_{k, i \pm}\left(x_{i \pm 1}\right)=0, \quad \text { for } l=0,1, \ldots, K
\end{aligned}
$$

where $D_{x}$ is the derivative operator in $x$, and $D_{x}^{0}=1$. For a uniform grid system the basis function satisfies the translational relation $\phi_{k, i}(x)=\phi_{k, i-n}\left(x-x_{n}\right)$.

In particular, the interpolating function in $\left[x_{i}, x_{i+1}\right]$ for the CIP-BS ${ }^{1}$ method is written by Hermite type interpolation as $f(x)=c_{0}+c_{1} \bar{x}+c_{2} \bar{x}^{2}+c_{3} \bar{x}^{3}$, where

$$
\begin{aligned}
& c_{0}=f_{i}, \quad c_{1}=f_{i}^{\prime}, \\
& c_{2}=3\left(f_{i+1}-f_{i}\right) / \Delta x^{2}-\left(2 f_{i}^{\prime}+f_{i+1}^{\prime}\right) / \Delta x, \\
& c_{3}=2\left(f_{i}-f_{i+1}\right) / \Delta x^{3}+\left(f_{i}^{\prime}+f_{i+1}^{\prime}\right) / \Delta x^{2},
\end{aligned}
$$

and $\bar{x}=x-x_{i}, \Delta x=x_{i+1}-x_{i}$. The coefficients $c_{0}, c_{1}, c_{2}$, $c_{3}$ are the same as those of the CIP method determined with the constraints: $f\left(x_{i}\right)=f_{i}, f^{\prime}\left(x_{i}\right)=f_{i}^{\prime}, f\left(x_{i+1}\right)=f_{i+1}$, $f^{\prime}\left(x_{i+1}\right)=f_{i+1}^{\prime}$.

The first derivative of the basis function is expressed as $D_{x} \phi_{k, i}(x)=\theta_{i-1, i} \phi_{k, i-}^{\prime}(x)+\theta_{i, i+1} \phi_{k, i+}^{\prime}(x)$. Here, we have used the fact that $\phi_{k, i \pm}(x) \delta\left(x-x_{i \pm 1}\right)=0$ due to the relation $x \delta(x)=0$, and $\phi_{k, i-}\left(x_{i}\right)=\phi_{k, i+}\left(x_{i}\right)$, where $\delta(x)$ is the Dirac delta function. Similarly, we can obtain the $l$-th order derivatives of $\phi_{k, i}(x)$ for $l \leq K+1$ as $D_{x}^{l} \phi_{k, i}(x)=$ $\theta_{i-1, i} \phi_{k, i-}^{(l)}(x)+\theta_{i, i+1} \phi_{k, i+}^{(l)}(x)$. Although the basis functions are constructed by using distribution functions, the functions represented in the CIP-BS ${ }^{K}$ method belong to the $\mathrm{C}^{K}$ class. Therefore, it is easily found that the $k$-th spatial derivative of $f(x)$ at the grid point $x_{i}$ equals the coefficient 
$f_{i}^{(k)}$, i.e. $\left.D_{x}^{k} f(x)\right|_{x=x_{i}}=f_{i}^{(k)}$. We can say that the basis set belongs to a complete set in the sense that the expansion (1) could represent the exact solution with any degree of accuracy in the limit $N \rightarrow \infty$ or $K \rightarrow \infty$. If $f(x)=0$ in Eq. (1), we can deduce that all the coefficients $f_{i}^{(k)}$ are zero, and that the basis functions are linearly independent. Then the function $f(x)$ can also be represented by this basis set as $\boldsymbol{f}=\left(\boldsymbol{f}_{1}, \boldsymbol{f}_{2}, \cdots, \boldsymbol{f}_{N}\right)$ where $\boldsymbol{f}_{i}=\left(f_{i}^{(0)}, f_{i}^{(1)}, \cdots, f_{i}^{(K)}\right)$.

Let us define addition and multiplication of the functions as follows:

$$
f(x)+g(x) \Leftrightarrow \boldsymbol{f}+\boldsymbol{g}=\left(\boldsymbol{f}_{1}+\boldsymbol{g}_{1}, \boldsymbol{f}_{2}+\boldsymbol{g}_{2}, \cdots, \boldsymbol{f}_{N}+\boldsymbol{g}_{N}\right)
$$

$$
\begin{aligned}
& c f(x) \Leftrightarrow c \boldsymbol{f}=\left(c \boldsymbol{f}_{1}, c \boldsymbol{f}_{2}, \cdots, c \boldsymbol{f}_{N}\right) \\
& f(x) \cdot g(x) \Leftrightarrow \boldsymbol{f} \cdot \boldsymbol{g}=\left(\boldsymbol{f}_{1} \cdot \boldsymbol{g}_{1}, \boldsymbol{f}_{2} \cdot \boldsymbol{g}_{2}, \cdots, \boldsymbol{f}_{N} \cdot \boldsymbol{g}_{N}\right),
\end{aligned}
$$

where $c$ is a scalar value. Addition and scalar multiplication for $f_{i}$ are

$$
\begin{aligned}
& \boldsymbol{f}_{i}+\boldsymbol{g}_{i}=\left(f_{i}^{(0)}+g_{i}^{(0)}, f_{i}^{(1)}+g_{i}^{(1)}, \cdots, f_{i}^{(K)}+g_{i}^{(K)}\right) \\
& c f_{i}=\left(c f_{i}^{(0)}, c f_{i}^{(1)}, \cdots, c f_{i}^{(K)}\right)
\end{aligned}
$$

and multiplication is given by

$$
\boldsymbol{f}_{i} \cdot \boldsymbol{g}_{i}=\left(h_{i}^{(0)}, h_{i}^{(1)}, \cdots, h_{i}^{(K)}\right),
$$

where $h_{i}^{(j)}=\sum_{l=0}^{j} \frac{j !}{l !(j-l) !} f_{i}^{(l)} g_{i}^{(j-l)}$. The identity of addition and multiplication are $(0,0, \cdots)$ and $(1,0, \cdots)$, respectively. Equations (8)-(10) are the same as the definition for differential algebra ${ }_{K} D_{1}$, (see Ref. (9)). Therefore, the functions $f^{-1}(x), \sqrt{f(x)}, \sin (f(x))$, or $\exp (f(x))$ can be uniquely represented by the basis set using the representation of $f(x)$. For Example, $f^{-1}(x)$ can be expressed as $\left(\frac{1}{f_{i}^{(0)}},-\frac{f_{i}^{(1)}}{f_{i}^{(0)^{2}}}, \frac{2 f_{i}^{(1)^{2}}-f_{i}^{(0)} f_{i}^{(2)}}{f_{i}^{(0)^{3}}}\right)$ when $K=2$ and $f_{i}^{(0)} \neq 0$. However, it is worth noting that, although the operation $D_{x}^{n}$ maps ${ }_{K} D_{1}$ into ${ }_{K-n} D_{1}$ in the differential algebra, we represent $D_{x}^{n}$ as a matrix by introducing the scalar product of the basis function $\phi_{k, i}(x)$ and $\phi_{k^{\prime}, i^{\prime}}(x)$ in the domain $R$ as ${ }^{(8)}$

$$
\left\langle\phi_{k, i} \mid \phi_{k^{\prime}, i^{\prime}}\right\rangle \equiv \int_{R} \phi_{k, i}(x) \phi_{k^{\prime}, i^{\prime}}(x) d x .
$$

Partial differential equations $\partial f(x, t) / \partial t=L[f(x, t)]$, where $L$ is a linear or nonlinear operator, are reduced to ordinary differential equations by the scalar product. Applying $\left\langle\phi_{k, i}\right|,(k=0,1,2, \cdots, K, i=0,1,2, \cdots, N)$, to the left of the equation, we obtain

$$
S \frac{d f}{d t}=L[f],
$$

where $S$ is a positive-definite matrix with the element $S_{k i, k^{\prime} i^{\prime}}=\left\langle\phi_{k, i} \mid \phi_{k^{\prime}, i^{\prime}}\right\rangle$. And $L$ is an $m+1$ dimensional matrix with the element

$$
L_{k i, k_{1} i_{1}, \cdots, k_{m} i_{m}}=\left\langle\phi_{k, i} \mid D_{x}^{n_{1}} \phi_{k_{1} i_{1}} \cdots D_{x}^{n_{m}} \phi_{k_{m} i_{m}}\right\rangle,
$$

where $m$ is a power of $f$ and $D_{x}^{n_{l}}$ is a differential operator on the $l$-th $f$. For example, if $L$ contains a term $f \partial f / \partial x$, the element of the corresponding matrix is $L_{k i, k_{1} i_{1}, k_{2} i_{2}}=$ $\left\langle\phi_{k, i} \mid \phi_{k_{1} i_{1}} D_{x} \phi_{k_{2} i_{2}}\right\rangle$. Since $S_{k i, k^{\prime} i^{\prime}}$ is non-zero only for $i^{\prime}=$ $i-1, i, i+1, S$ is a band diagonal matrix with bandwidth $3(K+1)$. The non-zero elements of the matrix representation of the operator $L$ are only for $i_{l}=i-1, i, i+1$, and can be analytically calculated. The rank of the differential operator must satisfy one of the following conditions: (1) $n_{l} \leq K$ for $l=1,2, \cdots, m$ (2) the maximum of $n_{l}$ is $K+1$, and the other $n_{l}$ are less than $K$ (3) the maximum of $n_{l}$ is $K+2$, and the other $n_{l}$ are less than $K-1$. Otherwise, terms like $\theta(x) \delta^{\prime}(x)$, which cannot be regularized, would appear. This procedure is equivalent to the one in the Galerkin method in which the residual $\frac{\partial}{\partial t} f(x, t)-L[f(x, t)]$ is required to be orthogonal to the basis functions $\phi_{k, i}(x)$. Roughly speaking, the subset of the equations resulting from the multiplication of $\left\langle\phi_{k, i}\right|$ corresponds to the equation $\partial f^{k} / \partial t=\partial^{k} L[f] / \partial x^{k}$.

\section{Numerical Results}

Here, we solve the continuity, Burgers, KdV, and hydrodynamic equation to demonstrate the effectiveness and accuracy of the CIP-BS method. For simplicity, we consider one-dimensional problems with a uniform grid. Furthermore, the grid system is fixed throughout the simulations. The matrices, $S$ and $L$, are calculated only at the beginning. If we want to change the grid system adaptively, the matrices must be re-calculated and the values and spatial derivatives on the grid points must be projected by using the approximating equation (Eq. (1)). The extension to a non-uniform grid system is straightforward, and multidimensional basis sets are discussed in the next section. The implicit solver with general sparse Jacobian matrices developed by Hindmarsh and Balsdon ${ }^{(10)}$ is used to time propagate the discretized equations (Eq. (12)).

\subsection{Continuity equation}

To show the fundamental capability of the CIP-BS method, we solve the continuity equation:

$$
\frac{\partial f}{\partial t}+u \frac{\partial f}{\partial x}=-f \frac{\partial u}{\partial x}
$$

on the unit periodic interval with initial condition $f(x, 0)=$ $\sin (2 \pi x)$ within a velocity field $u(x)=2-\sin (2 \pi x)$. The velocity field provides both a compression and expansion ${ }^{(11)}$ returning it to the initial position after every $t=1 / \sqrt{3}$ as shown in Fig. 1. Despite the large deformation caused by the velocity field, the solutions are accurate and do not diverge. In this calculation, $u(x)$ is expanded by Eq. (1) and represented as $\boldsymbol{u}=\left(\boldsymbol{u}_{1}, \boldsymbol{u}_{2}, \cdots, \boldsymbol{u}_{N}\right)$, where $\boldsymbol{u}_{i}=$ $\left(u\left(x_{i}\right)\right),\left(u\left(x_{i}\right), u^{\prime}\left(x_{i}\right)\right)$, or $\left(u\left(x_{i}\right), u^{\prime}\left(x_{i}\right), u^{\prime \prime}\left(x_{i}\right)\right)$ for the CIPBS $^{0}$, CIP-BS $^{1}$, and CIP-BS ${ }^{2}$ method, respectively. The periodic boundary condition is implemented as $\boldsymbol{f}_{1}=\boldsymbol{f}_{N}$, i.e. $f_{1}^{(k)}=f_{N}^{(k)}$ for $k=0,1, \cdots, K$. The discretized equations are written as 


$$
\begin{aligned}
& S_{k i, k^{\prime} i^{\prime}} \frac{d f_{i^{\prime}}^{\left(k^{\prime}\right)}}{d t} \\
& =-L_{k i, i_{1} k_{1}, i_{2} k_{2}}^{(001)} u_{i_{1}}^{\left(k_{1}\right)} f_{i_{2}}^{\left(k_{2}\right)}-L_{k i, i_{1} k_{1}, i_{2} k_{2}}^{(001)} f_{i_{1}}^{\left(k_{1}\right)} u_{i_{2}}^{\left(k_{2}\right)},
\end{aligned}
$$

for $i=1,2, \cdots, N, k=0,1, \cdots, K$, where $L_{k i, i_{1} k_{1}, i_{2} k_{2}}^{(001)}=$ $\left\langle\phi_{k, i} \mid \phi_{k_{1} i_{1}} D_{x} \phi_{k_{2} i_{2}}\right\rangle$. The summations for $k^{\prime}, k_{1}, k_{2}$ are taken on $0,1, \cdots, K$, and those for $i^{\prime}, i_{1}, i_{2}$ on $i-1, i, i+1$. Hereafter, if a subscript appears twice in a term, this summation is assumed.

Figure 2 shows errors defined by $\sum_{i=1}^{N}\left|f_{\text {exac }, i}-f_{\text {calc }, i}\right| \mid$

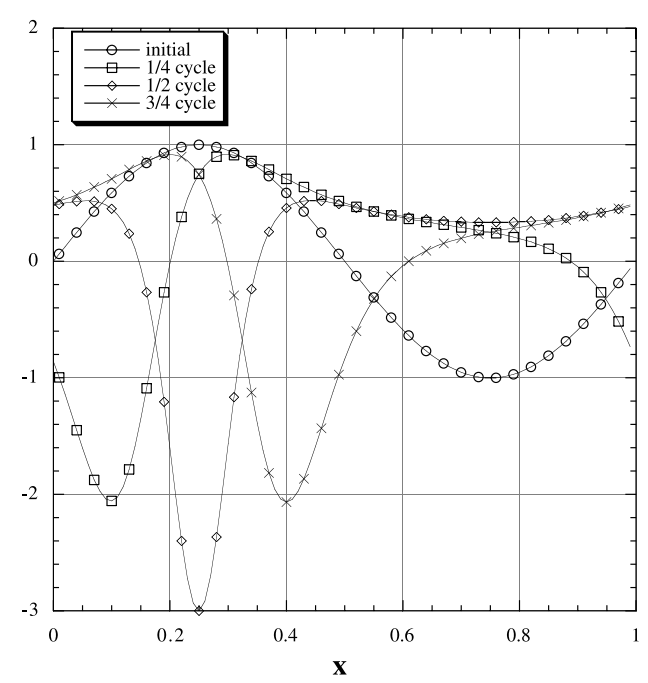

Fig. 1 The solutions of the continuity equation transporting an initial sine wave within a sinusoidal varying velocity field. CFL number $=0.1, \Delta x=0.01$, and the CIP-BS ${ }^{1}$ method is used. If there exists no error, the sine wave returns to the initial position after the $t=1 / \sqrt{3}=1$ cycle. The curves are identified in the legends.

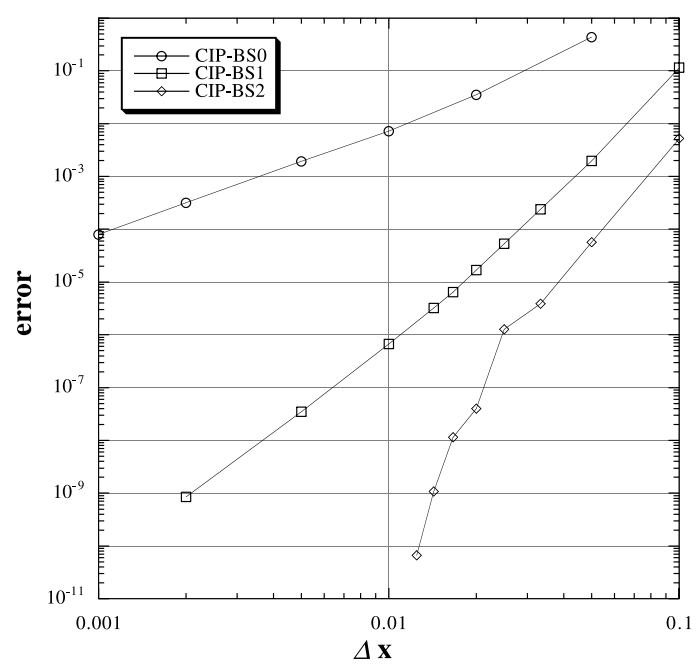

Fig. 2 The deviation from the exact solution (after 10 cycles) versus the grid interval for the solution of the continuity equation. The orders of the CIP-BS method used are as indicated.
$\sum_{i=1}^{N}\left|f_{\text {exac }, i}\right|$, where $f_{\text {exac }, i}$ is the exact and $f_{\text {calc }, i}$ the calculated value at the grid point $i$ after 10 cycles. This result shows 2nd, 4th, and 6th order accuracy for the CIP-BS ${ }^{0}$, CIP-BS ${ }^{1}$, and CIP-BS ${ }^{2}$ method, respectively. Although a special device for conservation is not implemented in the CIP-BS method, the relative error of $\int f(x, t) d x$, where $f(x, t)$ is piece-wise polynomial approximated by Eq. (1), is within $10^{-14}$ for all calculations. It is noted that $\int_{\text {at the grid points. }} f(x, t) d x$ is not generally equal to the sum of the values

\subsection{Burgers equation}

Next, to prove the applicability of the CIP-BS method for the nonlinear equations, we solve the Burgers equation:

$$
\frac{\partial f}{\partial t}+f \frac{\partial f}{\partial x}=v \frac{\partial^{2} f}{\partial x^{2}}
$$

where $v$ is the diffusion coefficient, on the unit periodic interval with the initial condition $f(x, 0)=\sin (2 \pi x)$. In this condition, the shock generated at $x=1 / 2$ is weakened as time elapses due to the dissipation. The discretized equations are written as

$$
\begin{aligned}
& S_{k i, k^{\prime} i^{i}} \frac{d f_{i^{\prime}}^{\left(k^{\prime}\right)}}{d t} \\
& =-L_{k i, i_{1} k_{1}, i_{2} k_{2}}^{(001)} f_{i_{1}}^{\left(k_{1}\right)} f_{i_{2}}^{\left(k_{2}\right)}+v L_{k i, i_{1} k_{1}}^{(02)} f_{i_{1}}^{\left(k_{1}\right)},
\end{aligned}
$$

for $i=1,2, \cdots, N, k=0,1, \cdots, K$, where $L_{k i, i_{1} k_{1}}^{(02)}=$ $\left\langle\phi_{k, i} \mid D_{x}^{2} \phi_{k_{1} i_{1}}\right\rangle$.

Figure 3 shows errors for $v=1 / 100$ at $t=10$, where the exact solutions are obtained by using the Cope-Hopf transformation $^{(12)}$. In spite of the nonlinearity of the Burgers equation, we can confirm that the CIP-BS ${ }^{0}$,

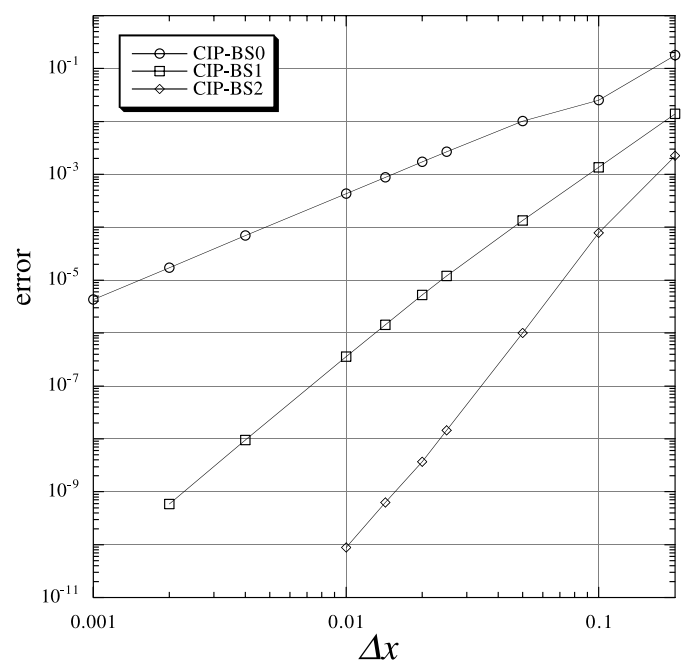

Fig. 3 The deviation from the exact solution at $t=10$ versus the grid interval for the solution of the Burgers equation on the unit periodic interval with the initial condition $f(x, 0)=\sin (2 \pi x)$. CFL number $=0.1, v=1 / 100$. The orders of the CIP-BS method used are as indicated. 


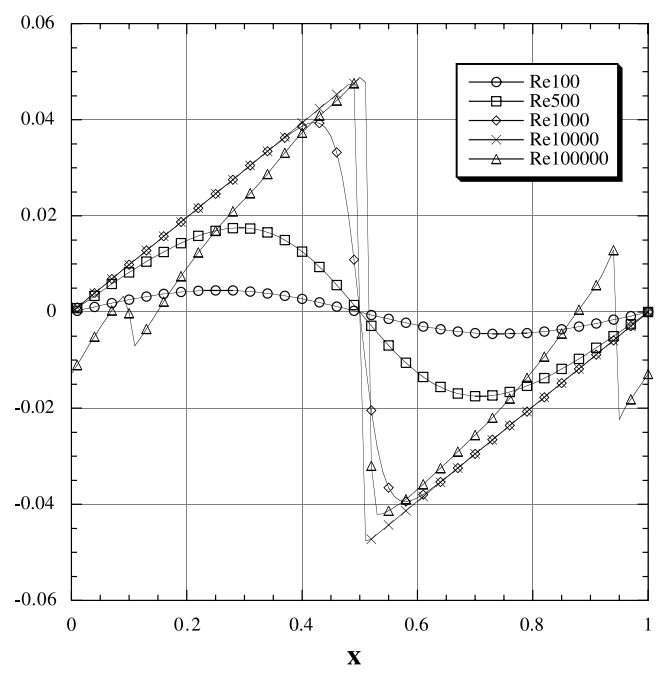

Fig. 4 The solution of the Burgers equation at $t=10$ on the unit periodic interval with the initial condition $f(x, 0)=$ $\sin (2 \pi x)$. CFL number $=0.1, \Delta x=0.01$, the CIP-BS ${ }^{1}$ method. The curves for $v=1 / R e$ are identified in the legends.

CIP-BS $^{1}$, and CIP-BS ${ }^{2}$ method have 2nd, 4th, and 6th order accuracy, respectively. Figure 4 shows solutions at $t=10$ when the diffusion coefficient is decreased, i.e. approaching the inviscid Burgers equation. As seen from Fig. 4, the solution largely degrade for $v=10^{-5}$. This situation is almost the same as those by the CIP-BS ${ }^{0}$ and CIP-BS ${ }^{2}$ method, because the maximum value of $\frac{\partial^{2} f}{\partial x^{2}}$ by the polynomial approximation is on the order of $\Delta x^{-2}$, however, the maximum value for the exact solution exceeds that for small $v$.

\subsection{KdV equation}

To illustrate that the CIP-BS method can deal with higher derivatives with high accuracy, we solve the KdV equation:

$$
\frac{\partial f}{\partial t}+f \frac{\partial f}{\partial x}=-\mu \frac{\partial^{3} f}{\partial x^{3}},
$$

on the two length periodic interval with the initial condition $f(x, 0)=\cos \pi x$ and the dispersion coefficient $\mu=$ $0.022^{2}$. This problem was first calculated by Zabusky and $\mathrm{Kruskal}^{(13)}$, using the finite differencing method where the momentum is identically conserved. Here, we discretize the equation as follows:

$$
\begin{aligned}
& S_{k i, k^{\prime} i^{\prime}} \frac{d f_{i^{\prime}}^{\left(k^{\prime}\right)}}{d t} \\
& =-L_{k i, i_{1} k_{1}, i_{2} k_{2}}^{(001)} f_{i_{1}}^{\left(k_{1}\right)} f_{i_{2}}^{\left(k_{2}\right)}-\mu L_{k i, i_{1} k_{1}}^{(03)} f_{i_{1}}^{\left(k_{1}\right)},
\end{aligned}
$$

for $i=1,2, \cdots, N, k=0,1, \cdots, K$, where $L_{k i, i_{1} k_{1}}^{(03)}=$ $\left\langle\phi_{k, i} \mid D_{x}^{3} \phi_{k_{1} i_{1}}\right\rangle$.

Figure 5 depicts the temporal development of the wave form, and Fig. 6 gives the space-time trajectories of the solitons calculated by the CIP-BS ${ }^{1}$ method. The results in Ref. (13) are well reproduced. We can observe that

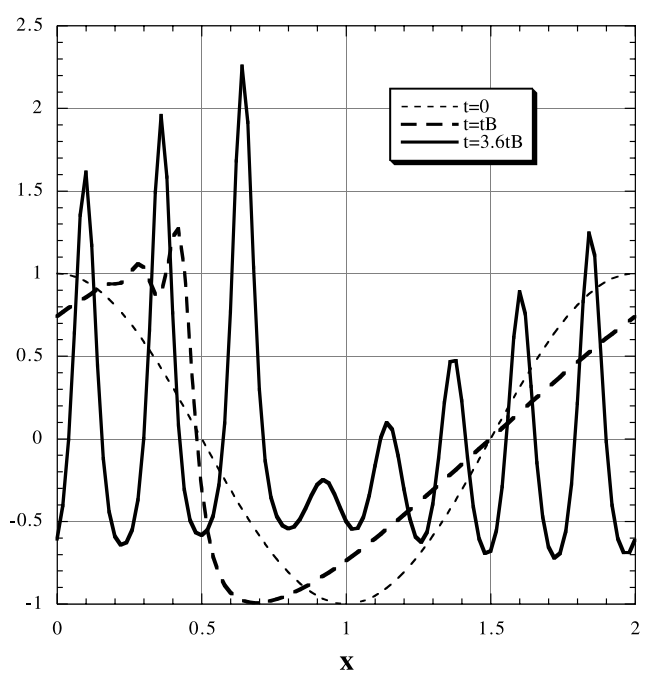

Fig. 5 The solution of the KdV equation by the CIP-BS ${ }^{1}$ method with an initial condition of $f(x, 0)=\cos \pi x$. CFL number $=0.1$, and $\Delta x=0.01$. The curves are drawn depending on the breakdown time of $t B=1 / \pi$.

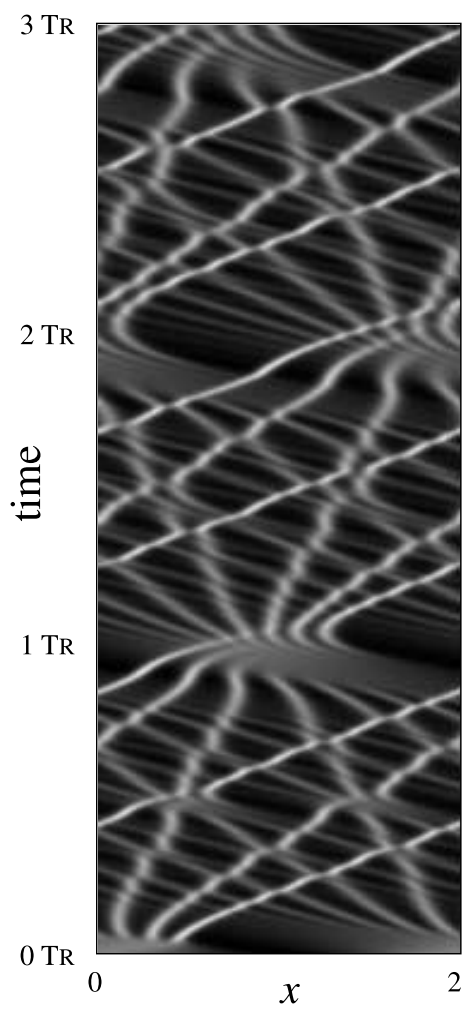

Fig. 6 Soliton trajectories in a space-time diagram. $T_{R}(=$ $30.4 t B=30.4 / \pi)$ is the recurrence time. The white and black color correspond to the value 3 and -1 , respectively.

the soliton retains its identity after strong nonlinear interactions with other solitons. In addition, all the solitons almost reconstruct the initial state after each recurrence time $T_{R}$. These calculations are carried out by the CIP$\mathrm{BS}^{1}$ or CIP-BS ${ }^{2}$ method, since the $\mathrm{KdV}$ equation contains 3rd order spatial derivatives and cannot be solved by the 
CIP-BS ${ }^{0}$ method as explained in the previous section. The quantity $\int f(x, t) d x$ is conserved for $0 \leq t \leq 3 T_{R}$ within $10^{-11}$ and $10^{-14}$ when solved by the CIP-BS ${ }^{1}$ and CIP-BS ${ }^{2}$ method, respectively.

\section{4 Hydrodynamic equation}

Finally, to reveal the ability to analyze multi-variable nonlinear hyperbolic equations, we solve shock tube problems by the hydrodynamic equations:

$$
\begin{aligned}
& \frac{\partial \rho}{\partial t}+u \frac{\partial \rho}{\partial x}=-\rho \frac{\partial u}{\partial x}, \\
& \frac{\partial u}{\partial t}+u \frac{\partial u}{\partial x}=-\frac{1}{\rho} \frac{\partial p}{\partial x}+\frac{\zeta}{\rho} \frac{\partial^{2} u}{\partial x^{2}}, \\
& \frac{\partial p}{\partial t}+u \frac{\partial p}{\partial x}=-\gamma p \frac{\partial u}{\partial x}+\zeta(\gamma-1)\left(\frac{\partial u}{\partial x}\right)^{2},
\end{aligned}
$$

where $\rho$ is the density, $u$ the velocity, $p$ the pressure, $\gamma$ the specific heat ratio, and $\zeta$ the artificial bulk viscosity. Since the CIP-BS method has low diffusion as shown in the solution of the Burgers and $\mathrm{KdV}$ equations, some artificial viscosity must be included. However, those introduced by Von Neumann ${ }^{(14)}$ or Nishiguchi ${ }^{(15)}$ are not convenient
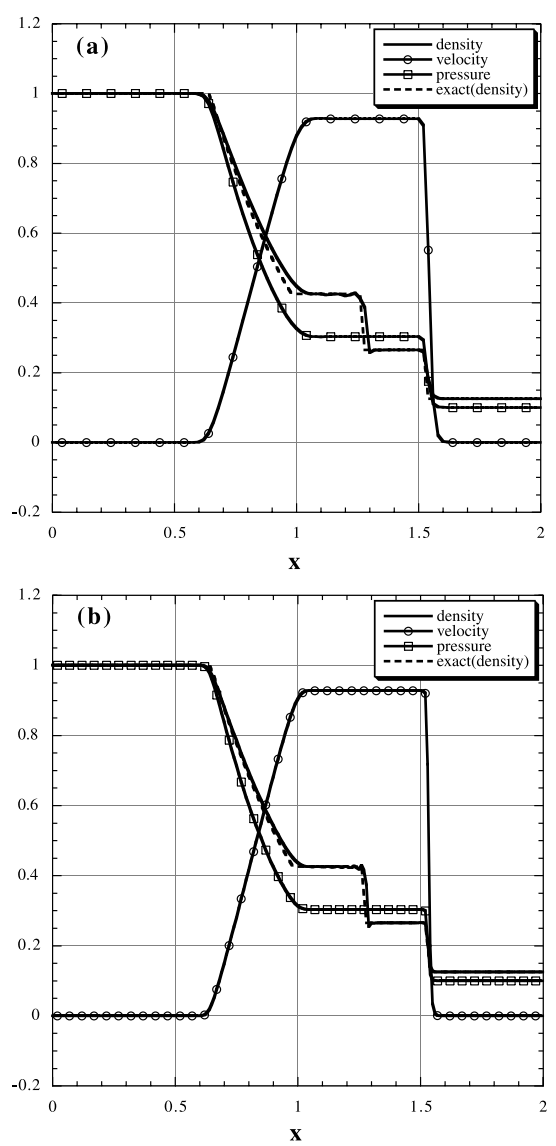

for our purpose due to their non-differentiable property. Therefore, we have introduced small real viscosity in the hydrodynamic equation. The value of $\zeta$ is determined so that the thickness of the shock becomes some multiple $\left(n_{s}\right)$ of the grid interval, i.e. $n_{s} \Delta x=\frac{8 \zeta}{(\gamma+1) \rho_{0}}$, where $\rho_{0}$ is the reference density and $n_{s}$ is set to 3 . The variables $\rho, u$, and $p$ are simultaneously expanded by Eq. (1) using the same basis set, and $\rho^{-1}$ appearing in the momentum equation is calculated by the differential algebra. The discretized equations are expressed as

$$
\begin{aligned}
& S_{k i, k^{\prime} i^{\prime}} \frac{d \rho_{i^{\prime}}^{\left(k^{\prime}\right)}}{d t} \\
& =-L_{k i, i_{1} k_{1}, i_{2} k_{2}}^{(001)} u_{i_{1}}^{\left(k_{1}\right)} \rho_{i_{2}}^{\left(k_{2}\right)}-L_{k i, i_{1} k_{1}, i_{2} k_{2}}^{(001)} \rho_{i_{1}}^{\left(k_{1}\right)} u_{i_{2}}^{\left(k_{2}\right)}, \\
& S_{k i, k^{\prime} i^{\prime}} \frac{d u_{i^{\prime}}^{\left(k^{\prime}\right)}}{d t}=-L_{k i, i_{1} k_{1}, i_{2} k_{2}}^{(001)} u_{i_{1}}^{\left(k_{1}\right)} u_{i_{2}}^{\left(k_{2}\right)} \\
& -L_{k i, i_{1} k_{1}, i_{2} k_{2}}^{(001)} \rho_{i_{1}}^{-1\left(k_{1}\right)} p_{i_{2}}^{\left(k_{2}\right)}+\zeta L_{k i, i_{1} k_{1}, i_{2} k_{2}}^{(002)} \rho_{i_{1}}^{-1^{\left(k_{1}\right)}} u_{i_{2}}^{\left(k_{2}\right)} \text {, } \\
& S_{k i, k^{\prime} i^{\prime}} \frac{d p_{i^{\prime}}^{\left(k^{\prime}\right)}}{d t}=-L_{k i, i_{1} k_{1}, i_{2} k_{2}}^{(001)} u_{i_{1}}^{\left(k_{1}\right)} p_{i_{2}}^{\left(k_{2}\right)}
\end{aligned}
$$
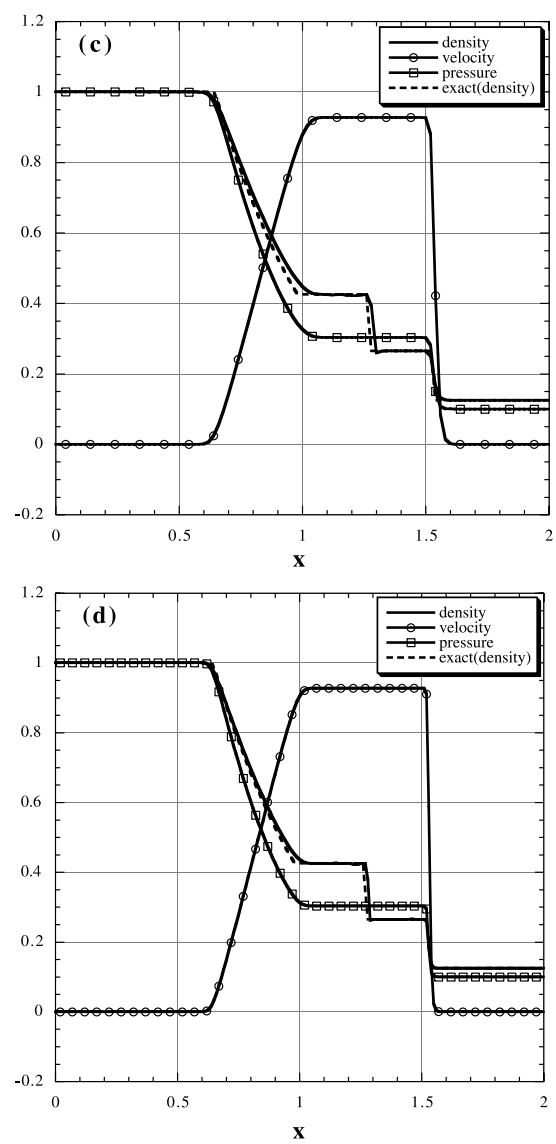

Fig. 7 Solutions of the weak shock problem. The initial conditions: $\rho=1, u=0, p=1$ for $0 \leq x \leq 1$, and $\rho=0.125, u=0, p=0.1$ for $1<x \leq 2$, where CFL number $=0.1, \gamma=1.4$. (a) $N=100$ by the CIP-BS ${ }^{1}$ method, (b) $N=200$ by the CIP-BS ${ }^{1}$ method, (c) $N=100$ by the CIP-BS ${ }^{2}$ method, (d) $N=200$ by the CIP-BS ${ }^{2}$ method. Profiles at $t=0.3$ are shown. The curves are identified in the legends. 

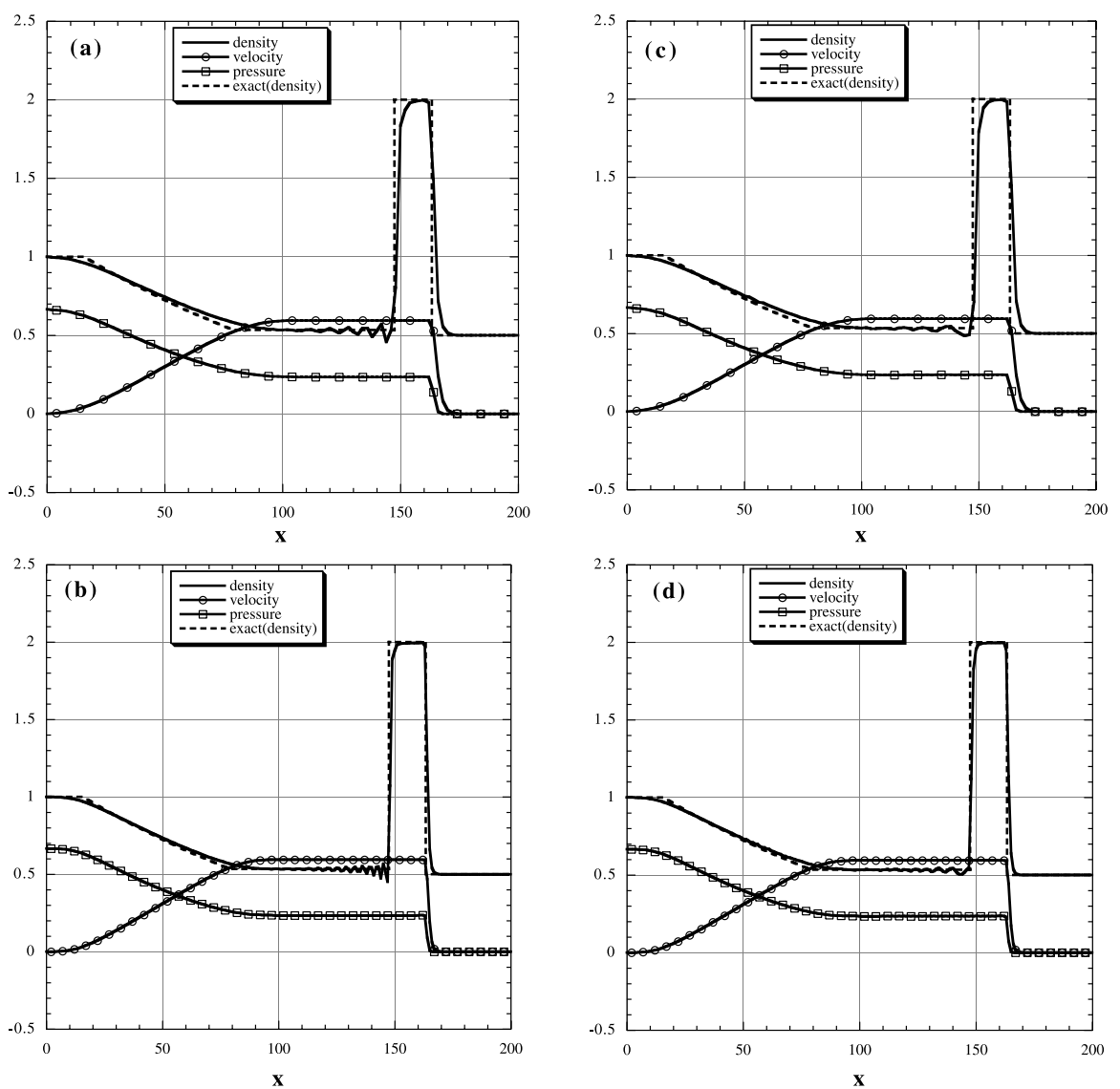

Fig. 8 Solutions of the strong shock problem. The initial conditions: $\rho=1, u=0, p=2 / 3$ for $0 \leq x \leq 100$, and $\rho=0.5, u=0, p=5 \times 10^{-5}$ for $100<x \leq 200$, where CFL number $=$ $0.1, \gamma=5 / 3$. (a) $N=100$ by the CIP-BS ${ }^{1}$ method, (b) $N=200$ by the CIP-BS ${ }^{1}$ method, (c) $N=100$ by the CIP-BS ${ }^{2}$ method, (d) $N=200$ by the CIP-BS ${ }^{2}$ method. Profiles at $t=80$ are shown. The curves are identified in the legends.

$-\gamma L_{k i, i_{1} k_{1}, i_{2} k_{2}}^{(001)} p_{i_{1}}^{\left(k_{1}\right)} u_{i_{2}}^{\left(k_{2}\right)}+\zeta(\gamma-1) L_{k i, i_{1} k_{1}, i_{2} k_{2}}^{(011)} u_{i_{1}}^{\left(k_{1}\right)} u_{i_{2}}^{\left(k_{2}\right)}$

for $i=1,2, \cdots, N, k=0,1, \cdots, K$, where $L_{k i, i_{1} k_{1}, i_{2} k_{2}}^{(011)}=$ $\left\langle\phi_{k, i} \mid D_{x} \phi_{k_{1} i_{1}} D_{x} \phi_{k_{2} i_{2}}\right\rangle$

Typical shock-tube problems are solved. Figure 7 shows the results for a weak shock problem with the initial conditions ${ }^{(16)}: \rho=1, u=0, p=1$ for $0 \leq x \leq 1$, and $\rho=0.125, u=0, p=0.1$ for $1<x \leq 2$. Figure 8 shows the results for a strong shock problem with the initial conditions $^{(16)}: \rho=1, u=0, p=2 / 3$ for $0 \leq x \leq 100$, and $\rho=0.5, u=0, p=5 \times 10^{-5}$ for $100<x \leq 200$. In these problems, the boundary values are fixed. Figure 9 shows the results for a shock collision problem with the initial conditions $^{(17)}: \rho=1, u=0, p=2 / 3$ for $x \leq 40, \rho=0.5$, $u=0, p=5 \times 10^{-5}$ for $40<x<280$ and $\rho=1.5, u=0$, $p=1$ for $280 \leq x \leq 400$. The reflecting boundary condition $\left(\frac{\partial \rho}{\partial x}=u=\frac{\partial p}{\partial x}=0\right)$, is imposed at both ends for this problem, i.e. $\rho_{1}^{(1)}=u_{1}^{(0)}=p_{1}^{(1)}=0, \rho_{N}^{(1)}=u_{N}^{(0)}=p_{N}^{(1)}=0$.

Our numerical simulations show that the CIP-BS method gives fairly good agreement with the analytical results, in particular, the correct shock speed and well re- solved shock front. It is emphasized that the results by Woodward and Colella ${ }^{(17)}$ for a shock collision problem are reproduced only with 200 grid points. Although small oscillations appear around the contact discontinuity for a strong shock problem, these do not diverge and smear out when we use finer meshes or a higher order method.

\section{Multi-Dimensional Basis Set}

It would be necessary to demonstrate multi-dimensional applications, to fully prove that the CIP-BS method is a general hyperbolic equation solver. Here, we briefly describe how the multi-dimensional basis set is constructed. We need a basis set where the multi-dimensional function is expressed as

$$
f(x)=\sum_{i=1}^{N} \sum_{k=0}^{K} f_{i}^{(k)} \phi_{k, i}(x)
$$

satisfying $\left.\frac{\partial^{k}}{\partial \boldsymbol{x}^{k}} f(\boldsymbol{x})\right|_{x=x_{i}}=f_{i}^{(\boldsymbol{k})}$, where $\boldsymbol{x}=\left(x_{1}, x_{2}, \cdots, x_{v}\right), \boldsymbol{i}=$ $\left(i_{1}, i_{2}, \cdots, i_{v}\right), \boldsymbol{N}=\left(N_{1}, N_{2}, \cdots, N_{v}\right), \boldsymbol{k}=\left(k_{1}, k_{2}, \cdots, k_{v}\right)$, and $v$ is the dimension of the space. It is easily verified that the functions

$$
\phi_{\boldsymbol{k}, i}(\boldsymbol{x})=\phi_{k_{1}, i_{1}}\left(x_{1}\right) \phi_{k_{2}, i_{2}}\left(x_{2}\right) \cdots \phi_{k_{v}, i_{v}}\left(x_{v}\right)
$$




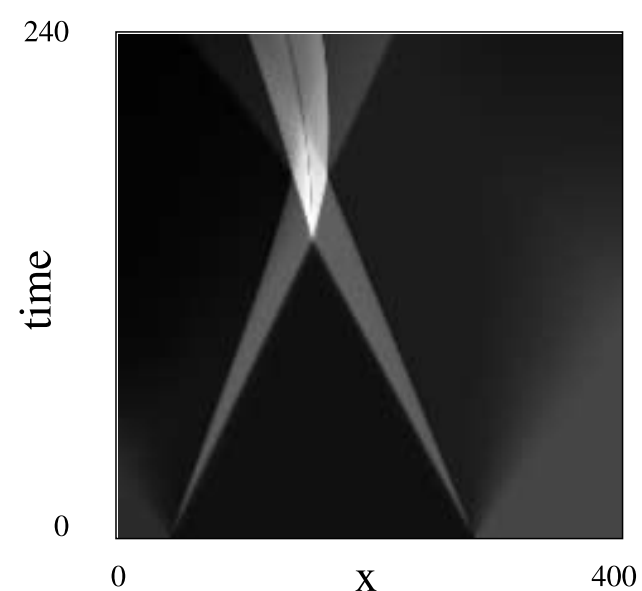

(a)

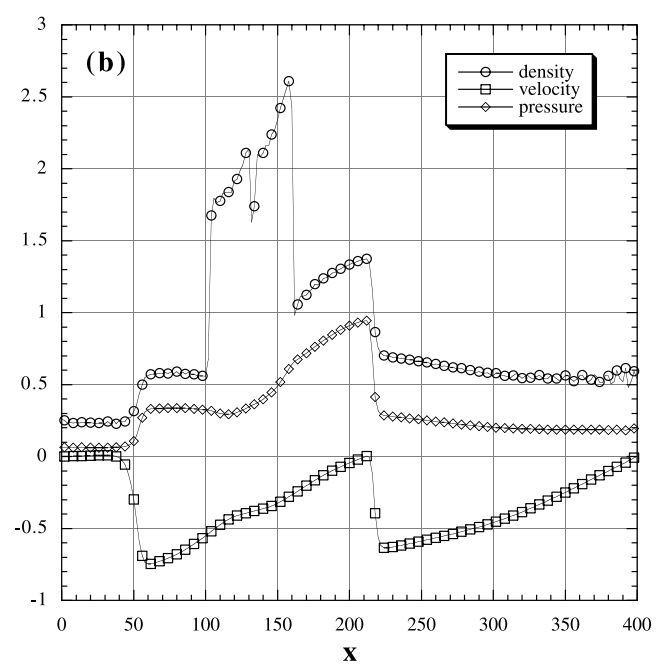

(b)

Fig. 9 Solutions of the shock collision problem. The initial conditions: $\rho=1, u=0, p=2 / 3$ for $x \leq 40, \rho=0.5$, $u=0, p=5 \times 10^{-5}$ for $40<x<280$ and $\rho=1.5, u=0$, $p=1$ for $280 \leq x \leq 400$, where the CFL number $=0.1$, $\gamma=5 / 3$, and $N=200$. The CIP-BS ${ }^{2}$ method is used. (a) Contours of the density in space-time, (b) density, velocity, and pressure are at $t=240$.

fulfill the requirements when Eqs. (2) and (3) are taken into account. For example, the two-dimensional interpolation function $f(x, y)$ within four grids $\left(x_{i}, y_{i}\right)-\left(x_{i+1}, y_{i}\right)$ $\left(x_{i+1}, y_{i+1}\right)-\left(x_{i}, y_{i+1}\right)$ is expressed as $f(x, y)=\sum_{n, m=0}^{3} c_{n m} x^{n} y^{m}$ for $K=1$, where

$$
\begin{aligned}
& c_{00}=f_{i j}^{(00)}, \quad c_{10}=f_{i j}^{(10)}, \quad c_{01}=f_{i j}^{(01)}, \\
& c_{20}=\left(-3 f_{i j}^{(00)}+3 f_{i+1 j}^{(00)}\right) / \Delta x^{2}-\left(2 f_{i j}^{(10)}+f_{i+1 j}^{(10)}\right) / \Delta x, \\
& c_{11}=0, \\
& c_{02}=\left(-3 f_{i j}^{(00)}+3 f_{i j+1}^{(00)}\right) / \Delta y^{2}-\left(2 f_{i j}^{(01)}+f_{i j+1}^{(01)}\right) / \Delta y, \\
& c_{30}=\left(2 f_{i j}^{(00)}-2 f_{i+1 j}^{(00)}\right) / \Delta x^{3}+\left(f_{i j}^{(10)}+f_{i+1 j}^{(10)}\right) / \Delta x^{2}, \\
& c_{21}=\left(-3 f_{i j}^{(01)}+3 f_{i+1 j}^{(01)}\right) / \Delta x^{2}, \\
& c_{12}=\left(-3 f_{i j}^{(10)}+3 f_{i j+1}^{(01)}\right) / \Delta y^{2},
\end{aligned}
$$

$$
\begin{aligned}
c_{03} & =\left(2 f_{i j}^{(00)}-2 f_{i j+1}^{(00)}\right) / \Delta y^{3}+\left(f_{i j}^{(01)}+f_{i j+1}^{(01)}\right) / \Delta y^{2}, \\
c_{31} & =\left(2 f_{i j}^{(01)}-2 f_{i+1 j}^{(01)}\right) / \Delta x^{3}, \\
c_{13} & =\left(2 f_{i j}^{(10)}-2 f_{i j+1}^{(10)}\right) / \Delta y^{3}, \\
c_{22} & =9\left(f_{i j}^{(00)}-f_{i j+1}^{(00)}-f_{i+1 j}^{(00)}+f_{i+1 j+1}^{(00)}\right) / \Delta x^{2} \Delta y^{2} \\
& +\left(6 f_{i j}^{(10)}-6 f_{i j+1}^{(10)}+3 f_{i+1 j}^{(10)}-3 f_{i+1 j+1}^{(10)}\right) / \Delta x \Delta y^{2} \\
& +\left(6 f_{i j}^{(01)}+3 f_{i j+1}^{(01)}-6 f_{i+1 j}^{(01)}-3 f_{i+1 j+1}^{(01)}\right) / \Delta x^{2} \Delta y, \\
c_{32} & =6\left(-f_{i j}^{(00)}+f_{i j+1}^{(00)}+f_{i+1 j}^{(00)}-f_{i+1 j+1}^{(00)}\right) / \Delta x^{3} \Delta y^{2} \\
& +\left(3 f_{i j}^{(10)}+3 f_{i j+1}^{(10)}-3 f_{i+1 j}^{(10)}+3 f_{i+1 j+1}^{(10)}\right) / \Delta x^{2} \Delta y^{2} \\
& +\left(-4 f_{i j}^{(01)}-2 f_{i j+1}^{(01)}+4 f_{i+1 j}^{(01)}+2 f_{i+1 j+1}^{(01)}\right) / \Delta x^{3} \Delta y, \\
c_{23} & =6\left(-f_{i j}^{(00)}+f_{i j+1}^{(00)}+f_{i+1 j}^{(00)}-f_{i+1 j+1}^{(00)}\right) / \Delta x^{2} \Delta y^{3} \\
& +\left(-4 f_{i j}^{(10)}+4 f_{i j+1}^{(10)}-2 f_{i+1 j}^{(10)}+2 f_{i+1 j+1}^{(10)}\right) / \Delta x \Delta y^{3} \\
& +\left(-3 f_{i j}^{(01)}-3 f_{i j+1}^{(01)}+3 f_{i+1 j}^{(01)}+3 f_{i+1 j+1}^{(01)}\right) / \Delta x^{2} \Delta y^{2}, \\
c_{33} & =4\left(f_{i j}^{(00)}-f_{i j+1}^{(00)}-f_{i+1 j}^{(00)}+f_{i+1 j+1}^{(00)}\right) / \Delta x^{3} \Delta y^{3} \\
& +2\left(f_{i j}^{(10)}-f_{i j+1}^{(10)}+f_{i+1 j}^{(10)}-f_{i+1 j+1}^{(10)}\right) / \Delta x^{2} \Delta y^{3} \\
& +2\left(f_{i j}^{(01)}+f_{i j+1}^{(01)}-f_{i+1 j}^{(01)}-f_{i+1 j+1}^{(01)}\right) / \Delta x^{2} \Delta y^{2} .
\end{aligned}
$$

It is easily verified $\left.\frac{\partial^{k_{x}} \partial^{k_{y}} f(x, y)}{\partial x^{k_{x}} \partial y^{k_{y}}}\right|_{(x, y)=\left(x_{i}, y_{j}\right)}=f_{i j}^{\left(k_{x} k_{y}\right)}$, $\left.\frac{\partial^{k_{x}} \partial^{k_{y}} f(x, y)}{\partial x^{k_{x}} \partial y^{k_{y}}}\right|_{(x, y)=\left(x_{i+1}, y_{j}\right)}=f_{i+1 j}^{\left(k_{x} k_{y}\right)},\left.\frac{\partial^{k_{x}} \partial^{k_{y}} f(x, y)}{\partial x^{k_{x}} \partial y^{k_{y}}}\right|_{(x, y)=\left(x_{i+1}, y_{j+1}\right)}$ $=f_{i+1 j+1}^{\left(k_{x} k_{y}\right)},\left.\frac{\partial^{k_{x}} \partial^{k_{y}} f(x, y)}{\partial x^{k_{x}} \partial y^{k_{y}}}\right|_{(x, y)=\left(x_{i}, y_{j+1}\right)}=f_{i j+1}^{\left(k_{x} k_{y}\right)}$ for $k_{x}, k_{y}=0,1$. And if coefficients $f_{i j}^{\left(k_{x} k_{y}\right)}$ are 1 only for $f_{00}^{(00)}, f_{01}^{(00)}, f_{11}^{(00)}$, $f_{01}^{(00)}$, then $f(x, y)=1$ holds within four grids $(0,0),(\Delta x, 0)$, $(\Delta x, \Delta y),(0, \Delta y)$. These are the desired properties for twodimensional interpolation functions.

Practical application of the multi-dimensional basis sets employing complex boundary conditions will be presented in the future.

\section{Conclusions}

We have generalized the CIP-BS method by introducing matrix representations and clarifying the relation with differential algebra to accommodate it to nonlinear partial differential equations. The linear and nonlinear partial differential equations are uniquely reduced to ordinary differential equations for values and spatial derivatives at the grid points. It is successfully applied to typical hyperbolic equations: the continuity equation, the Burgers equation, the Korteweg-de Vries equation, and one-dimensional hydrodynamic equations. It is proved that the method gives stable, less diffusive, and accurate results. Furthermore, since the matrices, $S$ and $L$, are sparse and sufficient to be calculated only at the beginning of the simulation, the method is computationally efficient. 


\section{Acknowledgment}

The authors would like to thank Prof. T. Tajima and Dr. T. Kimura at the Japan Atomic Energy Research Institute for their valuable discussion and encouragement.

\section{References}

( 1 ) Yabe, T. and Aoki, T., A Universal Solver for Hyperbolic Equations by Cubic-Polynomial Interpolation. I. One-Dimensional Solver, Comput. Phys. Commun., Vol.66 (1991), pp.219-232.

( 2 ) de Boor, C., A Practical Guide to Splines, (1978), Springer-Verlag, New York.

( 3 ) Yabe, T., Xiao, F. and Utsumi, T., The Constrained Interpolation Profile Method for Multiphase Analysis, J. Comput. Phys., Vol.169 (2001), pp.556-593.

( 4 ) Yabe, T., Ishikawa, T. and Wang, P.Y., A Universal Solver for Hyperbolic Equations by Cubic-Polynomial Interpolation. II. Two- and Three Dimensional Solvers, Comput. Phys. Commun., Vol.66 (1991), pp.233-242.

( 5 ) Yabe, T., A Universal Cubic Interpolation Solver for Compressible and Incompressible Fluids, Shock Waves, Vol.1 (1991), pp.187-195.

( 6 ) Yabe, T. and Xiao, F., Simulation Technique for Dynamical Evaporation Processes, Nucl. Eng. and Design, Vol.155 (1995), pp.45-53.

( 7 ) Yabe, T. and Xiao, F., Description of Complex and Sharp Interface with Fixed Grids in Incompressible and Compressible Fluids, Comput. Math. Appl., Vol.29 (1995), pp.15-25.

( 8 ) Utsumi, T., Yabe, T., Koga, J., Aoki, T. and Sekine, M.,
Accurate Basis Set by the CIP Method for the Solutions of the Schrödinger Equation, Comput. Phys. Commun., Vol.157 (2004), pp.121-138.

( 9 ) Berz, M., Arbitrary Order Description of Arbitrary Particle Optical Systems, Nucl. Instr. Meth. in Phys. Res., A, Vol.298 (1990), pp.426-440.

(10) Hindmarsh, A.C. and Balsdon, S., LSODE and LSODI, Two New Initial Value Ordinary Differential Equation Solvers, ACM-SIGNUM Newsletter, Vol.15, No.4 (1980), pp.10-11.

(11) Eastwood, J.W., Particle Simulation Methods in Plasma Physics, Comput. Phys. Commun., Vol.43 (1986), pp.89-106.

(12) Whitham, G.B., Linear and Nonlinear Waves, (1973), John Wiley \& Sons, NewYork.

(13) Zabusky, N.J. and Kruskal, M.D., Interaction of "Solitons" in a Collisionless Plasma and the Recurrence of Initial State, Phys. Rev. Lett., Vol.15, No.6 (1965), pp.240-243.

(14) Neumann, V. and Ritchtmyer, R.D., A Method for the Numerical Calculation of Hydrodynamic Shocks, J. Appl. Phys., Vol.21 (1950), pp.232-237.

(15) Nishiguchi, A. and Yabe, T., Second-Order Fluid Particle Scheme, J. Comput. Phys., Vol.52 (1983), pp.390413.

(16) Sod, G.A., A Survey of Several Finite Difference Methods for Systems of Nonlinear Hyperbolic Conservation Laws, J. Comput. Phys., Vol.27 (1978), pp.1-31.

(17) Woodward, P. and Colella, P., The Numerical Simulation of Two-Dimensional Fluid Flow with Strong Shocks, J. Comput. Phys., Vol.54 (1984), pp.115-173. 